\title{
CLASSIFIER-FREE DETECTION OF POWER LINE PYLONS FROM POINT CLOUD DATA
}

\author{
Mohammad Awrangjeb, Mohammad K. Islam \\ Institute for Integrated and Intelligent Systems, Griffith University, Nathan, QLD 4111, Australia \\ E-mail: \{m.awrangjeb, mohammadkhairul.islam\}@griffith.edu.au, Web: http://www.ict.griffith.edu.au/awrangjeb/
}

KEY WORDS: Power line extraction, transmission line extraction, pylon detection, power line reconstruction, LIDAR, point cloud data

\begin{abstract}
:
High density airborne point cloud data has become an important means for modelling and maintenance of a power line corridor. Since, the amount of data in a dense point cloud is huge even in a small area, an automatic detection of pylons in the corridor can be a prerequisite for efficient and effective extraction of wires in a subsequent step. However, the existing solutions mostly overlook this important requirement by processing the whole data into one go, which nonetheless will hinder their applications to large areas. This paper presents a new pylon detection technique from point cloud data. First, the input point cloud is divided into ground and nonground points. The non-ground points within a specific low height region are used to generate a pylon mask, where pylons are found stand-alone, not connected with any wires. The candidate pylons are obtained using a connected component analysis in the mask, followed by a removal of trees by comparing area, shape and symmetry properties of trees and pylons. Finally, the parallelism property of wires with the line connecting pair of candidate pylons is exploited to remove trees that have the same area and shape properties as pylons. Experimental results show that the proposed technique provides a high pylon detection rate in terms of completeness (100\%) and correctness $(100 \%)$.
\end{abstract}

\section{INTRODUCTION}

Reliable mapping of electrical power line corridors (PLCs) is important for a number of applications, such as monitoring of vegetation encroachment within a PLC (Ahmad et al., 2013) and analysis of power line structural stability (Guo et al., 2016). In fact, adding spatial context to asset (e.g., power line) management significantly improves the efficiency of managing an asset which involves millions of components, spread across thousands of linear kilometres and impacting diverse land uses. Traditionally, PLCs are surveyed in person or by manually inspecting aerial photos and videos. However, periodic manual inspection of thousands of kilometres of PLCs is not only time consuming and labour intensive, but also subject to errors due to involvement of human judgement. These inspection technologies are all spatially based, so a reliable map base is essential to gain maximum benefit. Furthermore, the map of most utilities may be out of date due to inaccuracies of original surveys and alterations to the network. Therefore, utility companies are seeking the benefits of modern technology to monitor the condition of their assets and reduce maintenance costs. Fortunately, the advent of airborne laser scanning technology, also known as LIght Detection And Ranging (LIDAR), has made the survey automatic and economic. By using the LIDAR technology, millions of 3D points on the earth surface and objects on it are collected and then processed off-line on powerful computers automatically. Thus, the LIDAR technology has made the entire survey more effective and efficient. A recent comprehensive review of various types of PLC surveying methods can be found in Matikainen et al. (Matikainen et al., 2016).

The processing of LIDAR data for 3D mapping of PLC has two main steps (McLaughlin, 2006): Points are first classified or segmented into different objects such as trees, pylons, wires and

${ }^{*}$ Corresponding author ground. Then, points on individual power line span are exploited to model wires between successive pylons. However, a well modelling of thin power lines requires a high density input data. Thus, there can be millions of points in a given $1 \mathrm{~km}^{2}$ scene. However, the actual number of points reflected from wires and pylons is far smaller than the number of input points. The detection of pylons can make the wire extraction step faster as in this case only the non-ground points between successive pylons need to be processed. Most existing power line extraction methods (e.g., McLaughlin (McLaughlin, 2006)) overlook the detection of pylons as a pre-requisite for power line extraction, except Sohn et al. (Sohn et al., 2012). Nonetheless, the absence of pylons can make a 3D PLC map incomplete and less useful.

In the literature, pylons are usually classified as a separate class using supervised classifiers, e.g., Random Forest (RF) (Kim and Sohn, 2013) and JointBoost (Guo et al., 2016). The classification output consists of a set of points for each class. Thus, 3D positions of individual pylons are not provided. Moreover, supervised classifiers have two main requirements: large training data set and balanced learning (Zhu and Hyyppä, 2014). A large training data set for pylons is in general hard to get for a given test scene. For example, the data set used by Kim and Sohn (Kim and Sohn, 2013) had only $0.81 \%$ points for pylons. So, pylons are in general a minority class compared to trees, buildings and wires in any input data. Using such an unbalanced classes of data, supervised classifiers tend to learn incorrect classification toward minority classes (Kim and Sohn, 2013).

Sohn et al. (Sohn et al., 2012) proposed the only pylon detection technique that provided individual pylon locations. 3D lines were first extracted using RANSAC (RANdom SAmple Consensus) algorithm. Then, these lines were converted into a binary image, from which 2D lines were classified using the RF classifier based on the orientation and parallelism properties. Finally, 


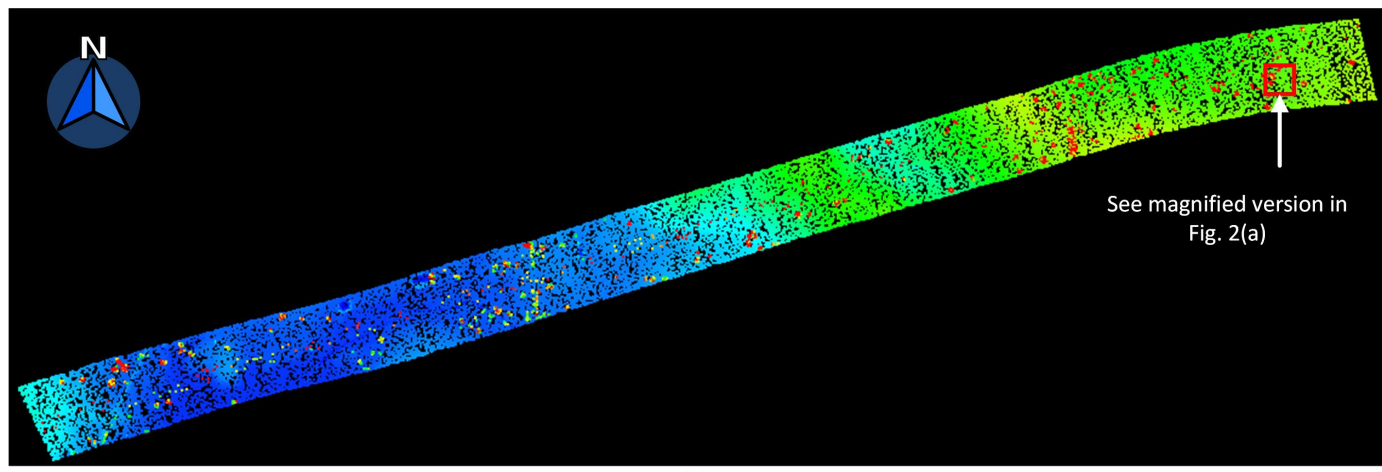

(a)

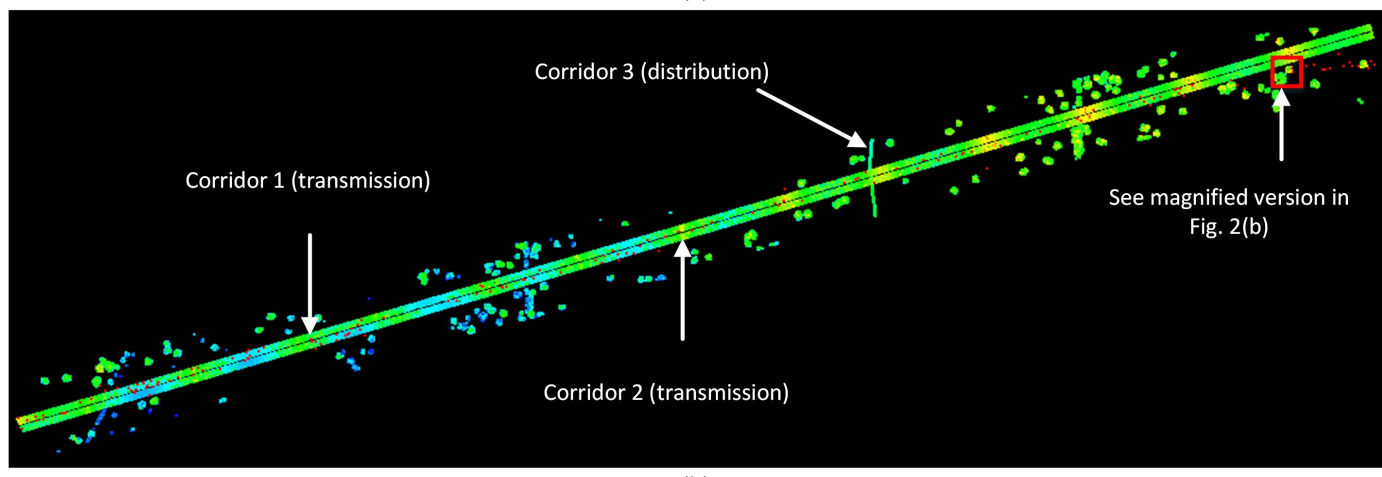

(b)

Figure 1. Test data set $(5,560 \mathrm{~m} \times 330 \mathrm{~m})$ : (a) input point cloud data and (b) non-ground points. ('N': north direction)

non-pylon objects were removed using a voting scheme based on contextual relations among the lines.

This paper proposes a new and classifier-free pylon detection technique from point cloud data. The proposed technique is based on the rules derived from the intuitive observation about the geometric property of objects in the scene. For instance, while pylons have a symmetric and/or cylindrical shape, trees mostly possess a random shape mainly due to branches and leaves. Since the proposed method does not use any supervised classifiers, it avoids the aforementioned problems associated with the classifier-based techniques.

\section{DATA SET}

Figure 1 shows the test data set from Maindample, Victoria, Australia. The test site is $5,560 \mathrm{~m}$ in length and $330 \mathrm{~m}$ in width. There are 3 power line corridors ( 2 transmissions and 1 distribution). The two transmission corridors include 26 pylons ( 13 on each), which are columns made of steel frames. The distribution corridor has only 2 poles, which are cylindrical columns. The width of each transmission and distribution corridors is $30 \mathrm{~m}$ and $10 \mathrm{~m}$, respectively. Note that the word 'pylon' is used for both 'pylon' and 'pole' in this paper.

The total number of points in the point cloud is $32,708,377$ (see Figure 1(a)). However, the number of non-ground points, shown in Figure 1(b), is 2,097,265 (16.5\% of the total points). Also, the number of non-ground points within the three corridors is only 279,867 ( $6.4 \%$ of the total points). Thus, the aim of the research presented in this paper is to automatically obtain all the pylons and, therefore, to effectively define the power line corridors to use in any future investigations.

\section{PROPOSED METHOD}

Figure 3 shows the flowchart of the proposed pylon detection technique. The input data consists of a LIDAR point cloud and DTM (Digital Terrain Model). The DTM is a height representation of the earth surface without any objects on it.

At first, the LIDAR points are classified into two groups: ground points, such as ground, road furniture and bushes that are below a height threshold, and non-ground points, which represent elevated objects (such as power lines, pylons and trees) above this threshold. The power line mask $M_{m}$, is generated using the nonground points. Individual pylons, power lines and trees are obtained as clusters of black pixels in $M_{m}$. Pylons and power lines of the same power line corridor are found connected in $M_{m}$, but if two or more corridors intersect (horizontally and/or vertically), then they are also found connected.

In $M_{m}$ wires and pylons are found connected and it is hard to separate them. So, the non-ground points are again processed to generate the pylon mask $M_{p}$, where pylons are found standalone and not connected at all with wires. The candidate pylons are obtained using a connected component analysis in the mask, followed by a removal of trees by comparing area, shape and symmetry properties of trees and pylons. Finally, the parallelism property of wires with the line connecting pair of candidate pylons is exploited to remove trees that have the same area and shape properties as pylons.

\subsection{Mask Generation}

If a DTM is not available, one can be generated from the LIDAR point cloud data using a commercial software. We assume that the DTM is given as an input to the proposed technique. Then, for each LIDAR point, the corresponding DTM height is used as the ground height, $H_{g}$. A height threshold $T_{h}=H_{g}+h_{c}$, where 


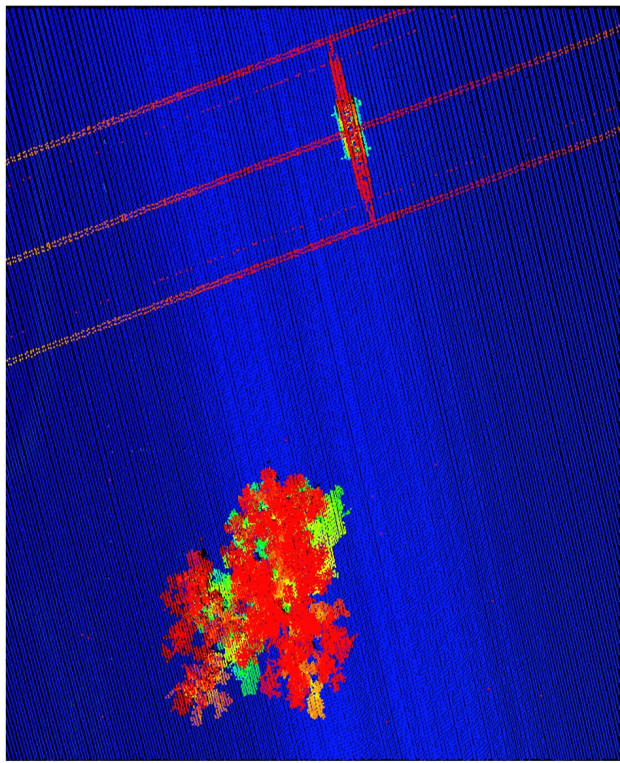

(a)

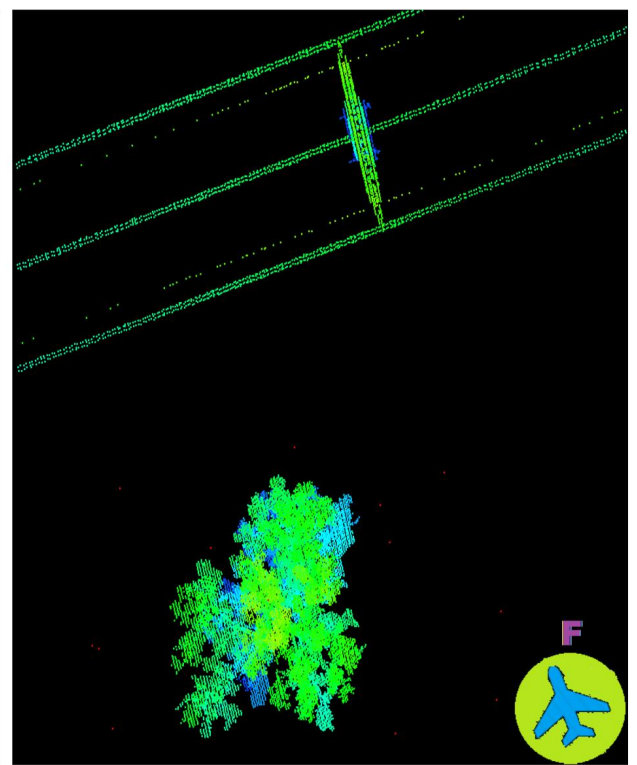

(b)

Figure 2. Sample scene from Figure 1: (a) all input points and (b) non-ground points. ('F': flight direction)

$h_{c}$ is a height constant that separates low height objects from high height objects, is then applied to the LIDAR data. Thus, the point cloud is divided into two groups: ground and non-ground points. The second group consists of the points that represent elevated objects, such as pylons, power lines and trees with heights above $T_{h}$. Many authors have used $h_{c}=2 \mathrm{~m}$ (Kim and Sohn, 2013) or $4 \mathrm{~m}$ (Zhu and Hyyppä, 2014). However, as we are focusing on the detection of individual pylons, which are sometimes only 10 to $15 \mathrm{~m}$ in height, to get all pylon points $h_{c}$ has been set at $1 \mathrm{~m}$ in this study. Figure 2(b) shows the non-ground points obtained from the input sample data shown in Figure 2(a).

The power line mask, $M_{m}$, as shown in Figure 4(b), is generated as follows. Let the resolution of $M_{m}$ be $R_{m}$ and all pixels are initially assigned 1 (white). Then, for each non-ground point the corresponding pixel in the mask is marked 0 (black). The value of $R_{m}$ is crucial here, because the input point cloud data can be sparse and/or there may not be enough points reflected from narrow wires to make the pylons on the same power line corridor connected. Ideally, in the mask there should be at least one wire between two consecutive pylons to make the whole corridor connected. However, the value of $R_{m}$ should not be large that will make the mask small in size and, thus, individual objects on the power line become too tiny and confusing in the mask. Consequently, the value of $R_{m}$ is kept fixed at $0.25 \mathrm{~m}$, but for each nonground point a neighbourhood $\Omega=N \times N$, where $N=2 n+1$ , is filled with 0 (black). The value of $n$ is estimated as follows:

$$
n=\left|\frac{R_{w}}{R_{m}}\right|-1
$$

where |.| indicates the round operation and $R_{w}$ is defined as

$$
R_{w}=\left\{\begin{array}{ll}
\frac{d_{m}}{2} & \text { if } d_{m}>1.0 \\
0.5 & \text { otherwise }
\end{array},\right.
$$

where $d_{m}$ is the maximum point-to-point distance on a wire. When there are two or more wires between the pylons in a corridor, it is fine to consider the smallest value of $d_{m}$ among the wires. The minimum value of $d_{m}$ is set at 0.5 in Eq. 2 . Therefore, $n \geq 1$, which ensures any pair of successive pylons in a corridor are connected. Figure 4(a) shows the mask with only the nonground point locations marked 0 . It is easily observed that there are a number of white pixels on each of the wires and so the different components of the power line corridor are not connected. However, after the neighbourhood filling individual components of a power line are found connected in the final mask shown in Figure 4(b).

As expected, $M_{m}$ contains pylons and wires connected. Thus, this mask is not suitable for detection of individual pylons. In order to find the pylons, a pylon mask $M_{p}$ is first generated using the same technique as presented above, but only non-ground points below a certain height $T_{H}$ are used. Given the minimum pylon's height is $15 \mathrm{~m}, T_{H}=12 \mathrm{~m}$ is set in order to avoid the connection between successive pylons. A power line is found in general in the form of a catenary curve and, therefore, its parts connected to the pylons have higher height (close to the pylon) than those away from the pylons. As a consequence, pylons are found stand-alone (without any connected wires) in $M_{p}$.

Since vertically some pylons may have holes in them, probably due to missing points, $M_{p}$ is then flood filled in order to remove the holes. Figure 4(c) shows the pylon mask for the sample scene. As can be observed, this mask contains a true pylon along with the trees in the scene. The single isolated points in the mask are due to noises in the data.

\subsection{Finding Candidate Pylons}

A connected component analysis is then carried out on $M_{p}$. Components that are larger than a predefined threshold $T_{a}$ are removed as trees. Since the largest length and width of a pylon are $20 \mathrm{~m}$ and $3 \mathrm{~m}$, respectively, $T_{a}=60 \mathrm{~m}^{2}$ is set in this investigation. Figure 4(d) shows 13 components in different colours. The largest component comprising a number of trees at the bottom of Figure 4(d) is removed at this stage.

Usually, trees and pylons possess different shape properties. For instance, majority of the points on a tree are reflected from branches and leaves, so there may be no or a very small number of points reaching its trunk. On the other hand, points are 


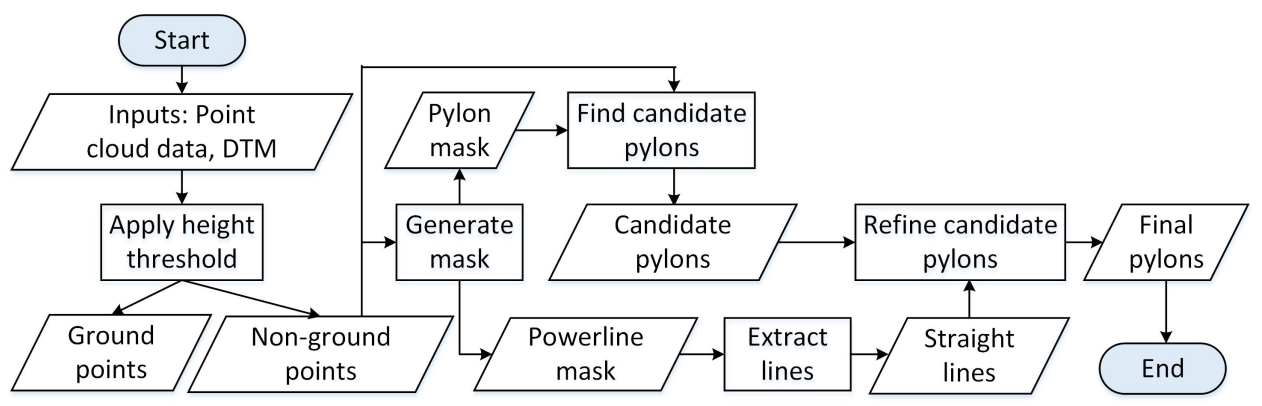

Figure 3. Flow chart of the proposed pylon detection technique.

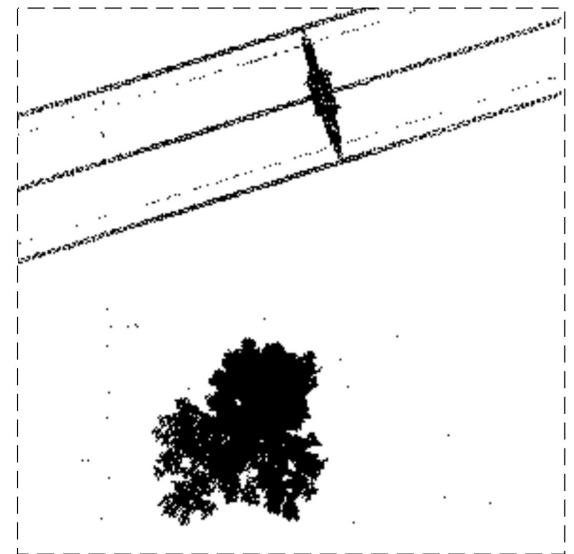

(a)

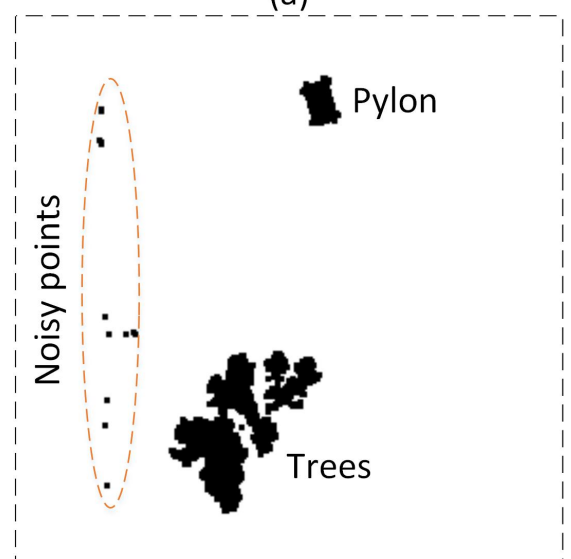

(c)

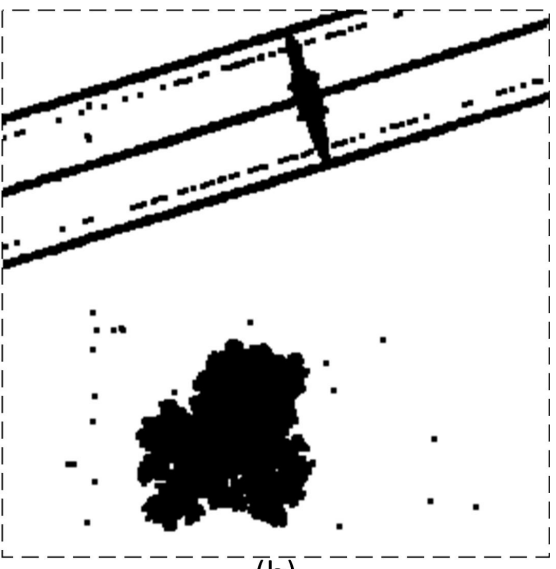

(b)

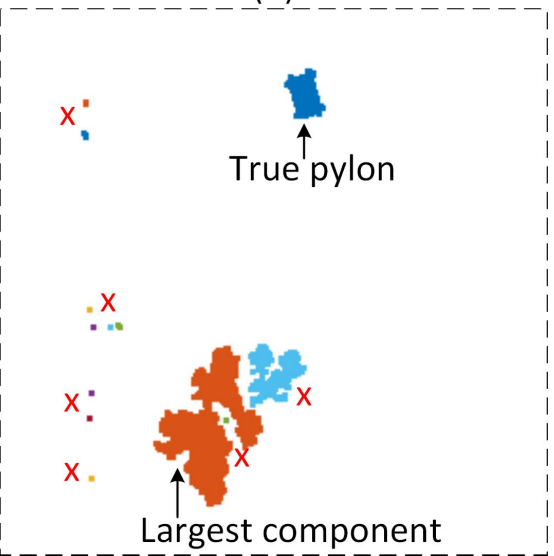

(d)

Figure 4. Generation of masks: (a) initial power line mask $M_{m}$, (b) final $M_{m}$, (c) Pylon mask $M_{p}$ and (d) Connected components in $M_{p}$. 


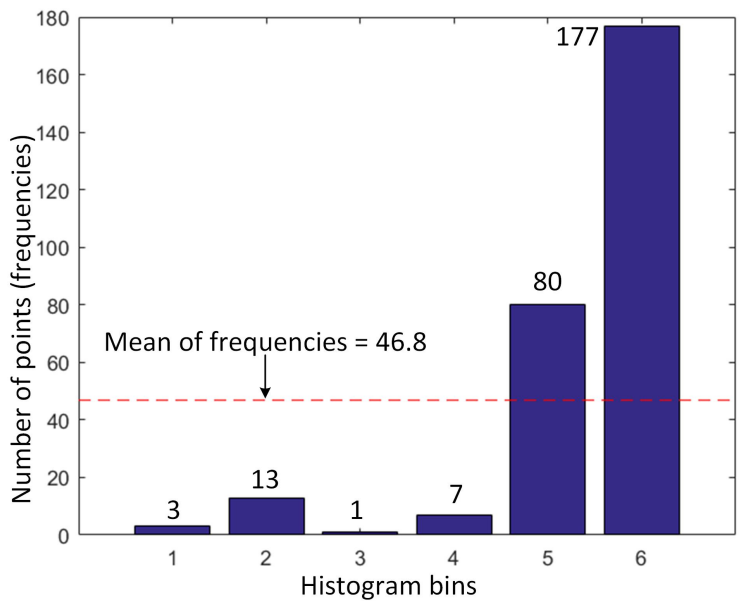

Figure 5. Removal of trees using a height histogram.

reflected from everywhere of a pylon. In addition, pylons can have at least two (horizontal) lines of symmetry, but a tree may not have any such lines of symmetry due to its random shape. Thus, while points on a tree make an asymmetric pattern, those of a pylon make a symmetric pattern.

Based on the above observation, the non-ground points within each of the remaining connected components are analysed as follows to remove the false pylons further. These points are first divided into six histogram bins at 2 m intervals: $2,4,6,8,10$ and 12 $\mathrm{m}$. Let the histogram of a component be $\left\{b_{i}\right\}$, where $1 \leq i \leq 6$ and $b_{i}$ indicates the number of points in bin $i$. If there are no points at any bins, the corresponding component is straightway removed as a tree. The isolated noisy points also get removed in this stage. For the remaining components, there are points at all six bins. Due to symmetric nature of a pylon the number of points in a bin does not differ significantly from other bins. In contrast, for a tree the number of points in a bin should differ significantly from other bins. Figure 5 shows the height histogram for a tree. As can be seen, the lower height bins (bin numbers 1 to 4 ) are for the trunk and contain much smaller numbers of points than the higher height bins (bin numbers 5 and 6). In order to remove trees having such a histogram pattern two ratios are estimated: $\left\{u_{i}\right\}=\frac{\left\{b_{i}\right\}}{m_{x}}$ and $\left\{v_{i}\right\}=\frac{\left\{b_{i}\right\}}{m_{n}}$, where $m_{x}$ and $m_{n}$ are maximum and mean values of $\left\{b_{i}\right\}$. If a connected component has a bin that has a very low number of points $\left(u_{i} \leq 0.1\right)$ or a very high number of points $\left(v_{i} \geq 2\right)$, then that component is removed as a tree. Figure 4(d) shows all 12 removed false pylons with nearby red crosses. The only remaining component is the true pylon in the sample scene.

\subsection{Finding Final Pylons}

Figure 6(a) shows all the candidate pylons in pink coloured squares after removal of majority of trees using the procedure discussed above. There are still some small trees, which exhibit similar characteristics of pylons, remain in the mask. In order to remove these trees the following inherent observation is applied.

There are long wires connecting successive pylons in a power line corridor. Nevertheless, such wires do not exist between trees. Therefore, straight lines are first extracted from the power line mask $M_{m}$ and then a constraint of having long straight lines in between nearby candidate pylons is applied.

To extract straight lines from $M_{m}$ the procedure introduced in (Awrangjeb et al., 2012) is followed. Canny edge algorithm is first applied to find edges. Corners are then detected on the extracted edges. A straight line is finally fitted between the two consecutive corners on each extracted edge. Figure 6(b) shows all the extracted lines $\left\{L_{k}\right\}$ in cyan colour, where $k \geq 1$, from the test data set. Thereafter, for each candidate pylon $P_{c}$, other candidate pylons $\left\{P_{o, j}\right\}$, where $j \geq 0$, in its vicinity (considering minimum and maximum distances between successive pylons $108 \mathrm{~m}$ and $450 \mathrm{~m}$, respectively) are obtained. If there are no neighbouring candidate pylons found (i.e., $\left\{P_{o, j}\right\}=\emptyset$ ), $P_{c}$ is removed as an isolated tree. Otherwise, for each $P_{n} \in P_{o, j}$ a line $P_{c} P_{n}$ is generated. All the long lines (at least $6 \mathrm{~m}$ ) from $\left\{L_{k}\right\}$ that are parallel to and reside within the vicinity (i.e., the power line corridor) of $P_{c} P_{n}$ are obtained. If there is no such lines exist between $P_{c}$ and $\forall P_{n} \in P_{o, j}$ then $P_{c}$ is removed as a tree.

Figure 6(b) shows all the pylons that survive after this stage in blue coloured squares and they are considered as the final pylons. The position of each of the pylon is the mean of the non-ground points used to form its connected component in $M_{p}$. The sequence of pylons in each of the three corridors (see Figure 1) can be easily obtained by considering which pylon is a neighbour of which in the above refinement procedure.

\section{EVALUATION, COMPARISON \& PARAMETERS}

To our knowledge this research is the second work that detects individual pylons from LIDAR point cloud data. Sohn et al. previously detected pylons as part of their power line scene classification and reconstruction method (Sohn et al., 2012). They classified the input point cloud data in several object types including pylons. The classification results were presented using the numbers of correctly and incorrectly classified points on pylons. Consequently, an existing performance evaluation result using the number of correctly and incorrectly detected pylons is absent in the state-of-the-art research. Moreover, since the data sets used in the existing research could not be accessed, an appropriate analysis of the comparative results between the proposed and existing techniques is therefore not possible in this investigation.

The proposed method has been implemented using MATLAB Version R2016a on a 64-bit Windows 7 Enterprise version machine with Intel(R) Core(TM) i7-6820HQ CPU @ 2.70GHz processor and 16 GB RAM. We have evaluated the performance using the object-based completeness and correctness, defined as follows (Awrangjeb and Fraser, 2014):

$$
\begin{gathered}
C_{m}=\frac{T P}{T P+F N} \text { and } \\
C_{r}=\frac{T P}{T P+F P},
\end{gathered}
$$

where $T P$ is the number of truly detected pylons, $F P$ is the number of falsely detected pylons and $F N$ is the number of missing pylons that cannot be detected by the involved pylon detection technique. Since, the proposed technique detected all the true pylons and did not detect any false pylons, both completeness and correctness are $100 \%$. The same performance was obtained by Sohn et al. (Sohn et al., 2012) using only 10 pylons. Due to the absence of any reference information about the detected pylons, their localisation error could not be estimated. However, in the magnified version, shown in Figure 6(b), each position is obtained at the centre of the pylon.

Regarding the use of different parameters by the proposed technique, most of the parameters, such as minimum and maximum 


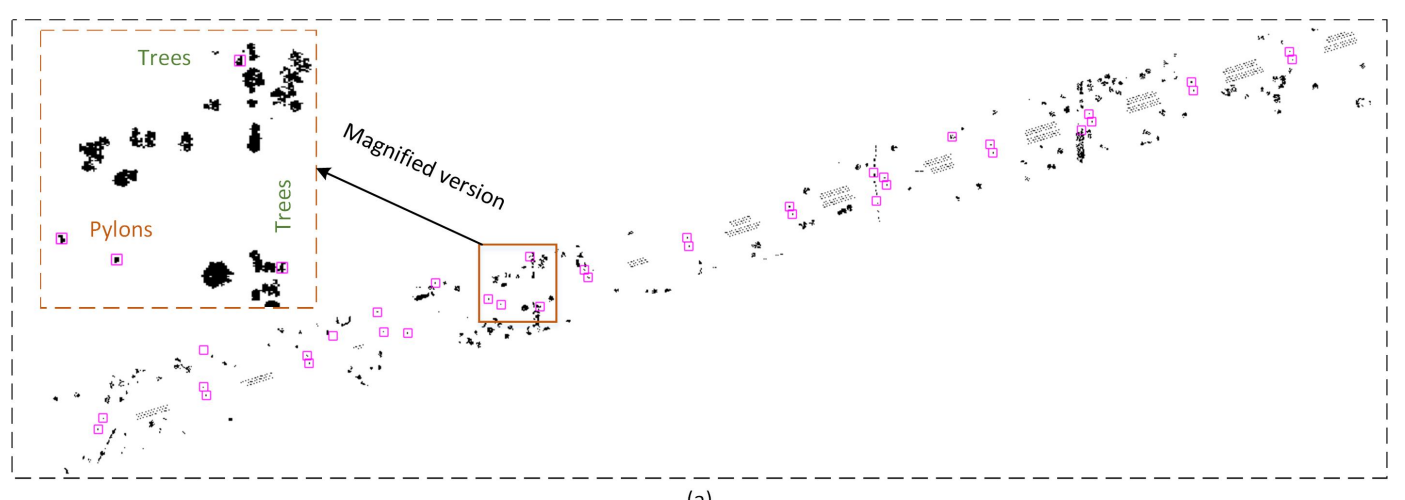

(a)

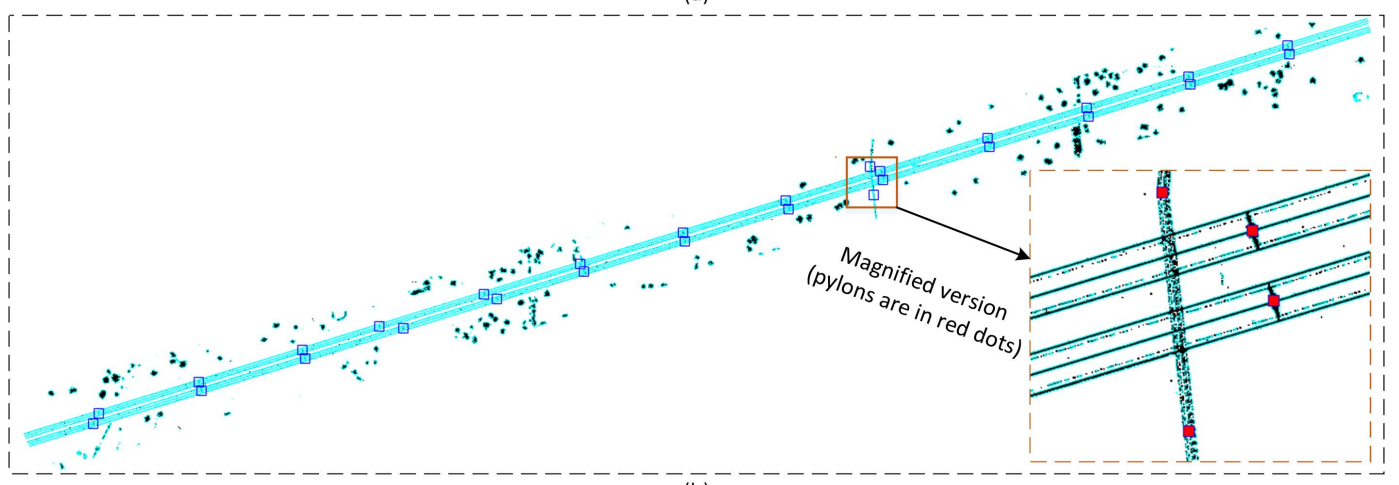

$(\bar{b})$

Figure 6. Refinement of candidate pylons in the whole test data set: (a) Candidate pylons and (b) Final pylons.

distances between pylons, are related to the input data and it is assumed that these parameter values are available with the data set. The resolution of the mask $R_{m}=0.25 \mathrm{~m}$ was previously used for building extraction (Awrangjeb and Fraser, 2014, Gilani et al., 2016). The height constant $h_{c}=1 \mathrm{~m}$ was earlier used in many research, including Awrangjeb et al. (Awrangjeb et al., 2012), on building detection. A higher value of $h_{c}$ can also be used since for the human safety purpose the power line wires are always hung at well above a certain height from the ground. However, if a higher $h_{c}$ value is used, the height range of 2 to $12 \mathrm{~m}$ above the ground for generation of the pylon mask $M_{p}$ needs to be updated as well.

The density of the input point cloud is 23.7 points $/ \mathrm{m}^{2}$. So, in order to keep $n \geq 1$ in Eq. 1, the maximum point-to-point distance $d_{m}$ is kept fixed at $0.5 \mathrm{~m}$. This value obviously would not make two close wires (say, within about $30 \mathrm{~cm}$ ) separate in the power line mask $M_{m}$. As evident from Figure 4(b), three pairs of close wires are captured as three thick lines in $M_{m}$.

The resolution of the input DTM is $1 \mathrm{~m}$. Since, the power line pylons are well above the ground (at least $15 \mathrm{~m}$ ), a fine (say, 0.5 m resolution) or a coarse (say, $3 \mathrm{~m}$ resolution) DTM should make no problem at all because the use of the DTM is simply to apply the (approximate) ground threshold $H_{g}$ value to obtain the non-ground point set. Moreover, the height constant $h_{c}=1 \mathrm{~m}$ reduces the effect of a coarse DTM. Even if some ground points may remain in the non-ground point set (specifically, in hilly areas) due to the use of a coarse DTM, this will have an insignificant effect since the pylon mask $M_{p}$ is generated from the non-ground points within 2 to $12 \mathrm{~m}$ above the ground.

Since, the proposed technique removes the huge amount of ground points in the mask generation step (Section 3.1), it makes the remaining steps very fast. Table 1 shows the running time for
Table 1. Running time for different steps of the proposed pylon detection technique.

\begin{tabular}{l|l|l|l}
\hline \hline Step & Section & Time (sec.) & Time (\%) \\
\hline Mask $M_{m}$ & 3.1 & 3.94 & 0.15 \\
Mask $M_{p}$ & 3.1 & 3.65 & 0.14 \\
Candidate pylons & 3.2 & 30.83 & 1.19 \\
Edge extraction from $M_{m}$ & 3.3 & 2474.09 & 95.27 \\
Line extraction from edges & 3.3 & 83.70 & 3.22 \\
Final pylons & 3.3 & 0.65 & 0.03 \\
\hline Total & & $\mathbf{2 5 9 6 . 8 6}$ & $\mathbf{1 0 0}$ \\
\hline \hline
\end{tabular}

different steps of the proposed pylon detection technique when it is applied to the large test data set $(5,560 \mathrm{~m} \times 330 \mathrm{~m})$ with more than 32.5 million points in the input point cloud data. For each step in the table the fastest time is recorded from about ten runs. As can be seen, most of the steps required a very short execution time (e.g., less than 5\% of the total running time). These steps are mostly newly proposed in this research.

However, the extraction of edges from the power line mask $M_{m}$ took a significant time (more than $95 \%$ of the total running time) because of the involved pixel-based edge extraction method adopted from Awrangjeb et al. (Awrangjeb et al., 2012). The size of $M_{m}$ was $21,736 \times 7,101$ pixels with resolution of $R_{m}=0.25 \mathrm{~m}$ for the whole test area. The majority of the time was mainly used to extract edges around the vegetation in the scene. The more the vegetation, the higher the edge extraction time. In order to reduce the edge extraction time, an efficient implementation of the edge extraction algorithm is required.

\section{CONCLUSION AND FUTURE WORK}

This paper has presented a new and classifier-free technique for pylon detection from point cloud data. Experimental results show 
that the proposed technique provides a high pylon detection rate. Since, it does not use any classification algorithms, it avoids the problem associated with classifier-based pylon extraction techniques mentioned in Section 1.

In our future investigation, we will extract the individual power line wires through processing the limited number of non-ground points that reside between successive pylons. A parallel computing for achieving a low computation cost is obvious as points inbetween a pair of neighbouring pylons can be independently processed. Our future work will also include 3D modelling of power line corridors, specifically each extracted wire will be modelled as a catenary curve (Ahmad et al., 2013). In addition, it would be interesting to investigate the performance of the proposed pylon detection technique with a low point density input data set. An empirical study with different value of $d_{m}$ (the maximum point-to-point distance) could be exploited for this investigation (Awrangjeb and Fraser, 2014).

\section{ACKNOWLEDGMENT}

The authors would like to thank the AAM Group (www. aamgroup.com) for providing the test data set. The project is supported by Griffith University's New Researcher Grant (036 Research Internal).

\section{REFERENCES}

Ahmad, J., Malik, A. S., Xia, L. and Ashikin, N., 2013. Vegetation encroachment monitoring for transmission lines right-ofways: A survey. ISPRS Journal of Photogrammetry and Remote Sensing 95(2), pp. 339-352.

Awrangjeb, M. and Fraser, C. S., 2014. Automatic segmentation of raw LIDAR data for extraction of building roofs. Remote Sensing 6(5), pp. 3716-3751.

Awrangjeb, M., Zhang, C. and Fraser, C. S., 2012. Building detection in complex scenes thorough effective separation of buildings from trees. Photogrammetric Engineering \& Remote Sensing 78(7), pp. 729-745.

Gilani, S. A. N., Awrangjeb, M. and Lu, G., 2016. Robust building roof segmentation using airborne point cloud data. In: IEEE International Conference on Image Processing, Phoenix, Arizona, USA, pp. 859-863.

Guo, B., Li, Q., Huang, X. and Wang, C., 2016. An improved method for power-line reconstruction from point cloud data. $R e$ mote Sensing 8(1), pp. 1-17.

Kim, H. B. and Sohn, G., 2013. Point-based classification of power line corridor scene using random forests. Photogrammetric Engineering \& Remote Sensing 79(9), pp. 821-833.

Matikainen, L., Lehtomäki, M., Ahokas, E., Hyyppä, J., Karjalainen, M., Jaakkola, A., Kukko, A. and Heinonen, T., 2016. Remote sensing methods for power line corridor surveys. ISPRS Journal of Photogrammetry and Remote Sensing 19(9), pp. 10 31 .

McLaughlin, R. A., 2006. Extracting transmission lines from airborne LIDAR data. IEEE Geoscience and Remote Sensing Letters 3(2), pp. 222-226.

Sohn, G., Jwa, Y. and Kim, H. B., 2012. Automatic powerline scene classification and reconstruction using airborne LIDAR data. ISPRS Annals of the Photogrammetry, Remote Sensing and Spatial Information Sciences I-3, pp. 167-172.
Zhu, L. and Hyyppä, J., 2014. Fully-automated power line extraction from airborne laser scanning point clouds in forest areas. Remote Sensing 6(11), pp. 11267-11282. 